EDITORIAL

\title{
Exciting times ahead for older patients with acute myeloid leukemia: azacitidine and venetoclax followed by allogeneic hematopoietic cell transplantation
}

(c) The Author(s), under exclusive licence to Springer Nature Limited 2021

Bone Marrow Transplantation (2022) 57:147-148; https://doi.org/ 10.1038/s41409-021-01474-9

Acute myeloid leukemia (AML) is a known disease of older persons, where the median age at diagnosis is 68 years [1]. Intensive chemotherapy, mainly $7+3$ regimen have been developed and evaluated mainly in younger adults aged 60 years or below. Patients older than 60 years historically performed poorly if treated with intensive chemotherapy, with reported high early mortality rates reaching 40\% [2-4]. Even among responders, their duration of response is limited and their median overall survival (OS) cannot exceed 6-8 months at best. Biological age is not the only culprit, as other factors also play a role in reduced survival, such the performance status at time of diagnosis, the presence of comorbidity conditions or organ dysfunction, and most importantly the biology of AML disease that usually is associated with high-risk cytogenetic or molecular aberrations.

Epigenetic therapy in older AML patients was considered the safer alternative to intensive chemotherapy where azacitidine or decitabine as single agent resulted in improvements in clinical outcomes with a median OS of 8-10 months [5, 6]. Hypomethylating agents (HMA) were the standard of care for this older population for the past decade. Until recently, the introduction of venetoclax, an orally available selective BCL2-inhibitor, has changed the AML treatment paradigm of older patients. The combination of HMA or low-dose cytarabine (LDAC) showed superior response rates and improvements in median OS in two randomized trials named the VIALE-A and VIALE-C trials $[7,8]$. The new regimen was approved by the FDA for newly diagnosed AML patients older than 75 years or unfit for intensive chemotherapy. More interestingly, venetoclax-based combinations were capable of inducing faster and deeper remissions, with median time to response of 1 month $[7,8]$. Furthermore, the overall toxicity profile of venetoclax combined with azacitidine or LDAC was acceptable; despite the increased risk of myelosuppression and febrile neutropenia in up to $40 \%$ of patients, early mortality was $<10 \%$ in both studies [7, 8]. These encouraging data rendered venetoclax-based combinations the new standard of care in older adults with newly diagnosed AML. However, although the majority of patients will respond to this new standard regimen, relapses still occur with more resistant clones. Those who lose their responses after HMA-venetoclax combinations will have a dismal outcome and a median OS of 2.4 months regardless of salvage treatment used [9]. Strategies to improve further the long-term outcomes of venetoclax-based combinations and reach a potential cure for older AML adults are much needed.

Nevertheless, allogeneic hematopoietic cell transplantation (allo-HCT) continues to be a cornerstone of AML curative therapy, especially for high-risk disease. Recent advances in conditioning regimens with the widespread use and experience in the reducedintensity conditioning $(\mathrm{RIC})$ allowed the delivery of allo-HCT to patients up to 70 years of age.

In this issue, Pollyea and his group are retrospectively reporting the clinical outcomes of allo-HCT following the combination treatment of azacitidine and venetoclax in patients with newly diagnosed AML [10]. They have included a total of 52 transplanteligible patients, of which 21 patients received allo-HCT, whereas the other 31 patients deferred allo-HCT mostly for personal reasons. Patients who were transplanted were relatively younger than those who deferred allo-HCT; however, both groups had similar comorbidity index scores $(p=0.58)$. After a median followup of 24 months from diagnosis, when comparing the OS between the two groups, patients treated with HMA and venetoclax combination as a bridge to allo-HCT had a significantly better OS (median not reached versus 17.2 months, $p=0.01$ ) compared to those who deferred allo-HCT. Among patients who had allo-HCT as consolidation, the 1-year cumulative incidence of relapse was reported at $20 \%$ with an acceptable 1-year transplantrelated mortality of $11 \%$ [10].

A 2-year OS of $76 \%$ among older patients who were transplanted is definitely an exciting finding, showing that older patients are now able to reach a long-term disease control and better survival once complete remission is achieved with less intensive less toxic combination regimen such as HMA and venetoclax.

Many novel agents are now under investigation to be combined with venetoclax aiming at overcoming resistance and improving clinical responses. Until then, older patients deserve to be assessed by a transplant specialist and determine the need for allo-HCT based on individual assessment including disease risk score, performance status and comorbidity index.

Furthermore, given the high efficacy and excellent tolerability of HMA and venetoclax induction combinations, there is a rationale to consider them in older fit patients aged $60-75$ years to induce deep remissions and maintain patients' good performance. This strategy serves as an excellent bridge to allo-HCT and potential cure.

Ali Bazarbachi ${ }^{1 凶}$
${ }^{1}$ Department of Internal Medicine, American University of Beirut
Medical Center, Beirut, Lebanon. ${ }^{\bowtie}$ email: bazarbac@aub.edu.lb

\section{REFERENCES}

1. Shallis RM, Wang R, Davidoff A, Ma X, Zeidan AM. Epidemiology of acute myeloid leukemia: recent progress and enduring challenges. Blood Rev. 2019;36:70-87.

2. Kantarjian H, O'Brien S, Cortes J, Giles F, Faderl S, Jabbour E, et al. Results of intensive chemotherapy in 998 patients age 65 years or older with acute myeloid 
leukemia or high-risk myelodysplastic syndrome: predictive prognostic models for outcome. Cancer. 2006;106:1090-8.

3. Kantarjian H, Ravandi F, O'Brien S, Cortes J, Faderl S, Garcia-Manero G, et al. Intensive chemotherapy does not benefit most older patients (age 70 years or older) with acute myeloid leukemia. Blood. 2010;116:4422-9.

4. Sasaki K, Ravandi F, Kadia TM, DiNardo CD, Short NJ, Borthakur G, et al. De novo acute myeloid leukemia: a population-based study of outcome in the United States based on the Surveillance, Epidemiology, and End Results (SEER) database, 1980 to 2017. Cancer. 2021;127:2049-61.

5. Dombret H, Seymour JF, Butrym A, Wierzbowska A, Selleslag D, Jang JH, et al. International phase 3 study of azacitidine vs conventional care regimens in older patients with newly diagnosed AML with $>30 \%$ blasts. Blood. 2015;126:291-9.

6. Kantarjian HM, Thomas XG, Dmoszynska A, Wierzbowska A, Mazur G, Mayer J, et al. Multicenter, randomized, open-label, phase III trial of decitabine versus patient choice, with physician advice, of either supportive care or low-dose cytarabine for the treatment of older patients with newly diagnosed acute myeloid leukemia. J Clin Oncol. 2012;30:2670-7.

7. DiNardo CD, Jonas BA, Pullarkat V, Thirman MJ, Garcia JS, Wei AH, et al. Azacitidine and venetoclax in previously untreated acute myeloid leukemia. $N$ Engl J Med. 2020;383:617-29.

8. Wei AH, Montesinos $\mathrm{P}$, Ivanov V, DiNardo CD, Novak J, Laribi K, et al. Venetoclax plus LDAC for newly diagnosed AML ineligible for intensive chemotherapy: a phase 3 randomized placebo-controlled trial. Blood. 2020;135:2137-45.
9. Maiti A, Rausch CR, Cortes JE, Pemmaraju N, Daver NG, Ravandi F, et al. Outcomes of relapsed or refractory acute myeloid leukemia after frontline hypomethylating agent and venetoclax regimens. Haematologica. 2021;106:894-8.

10. Pollyea DA, McMahon C, Schwartz M, Jordan CT, Rabinovitch R, Gutman JA. Venetoclax and azacitidine followed by allogeneic transplant results in excellent outcomes and may improve outcomes versus maintenance therapy among newly diagnosed AML patients older than 60. Bone Marrow Transplant. 2021.

\section{CONFLICT OF INTEREST}

The author declares no competing interests.

\section{ADDITIONAL INFORMATION}

Correspondence and requests for materials should be addressed to Ali Bazarbachi.

Reprints and permission information is available at http://www.nature.com/ reprints

Publisher's note Springer Nature remains neutral with regard to jurisdictional claims in published maps and institutional affiliations. 